\title{
Variabilidade espacial de atributos físicos do solo e seus efeitos sobre a produtividade do trigo ${ }^{1}$
}

\section{Spatial variability of soil physical attributes and its effects on wheat yield}

\author{
Ademir Natal Corrêa ${ }^{2}$; Maria Hermínia Ferreira Tavares ${ }^{3 *}$; \\ Miguel Angel Uribe-Opazo ${ }^{4}$
}

\section{Resumo}

Este trabalho teve como objetivo estudar a influência da variabilidade espacial dos atributos físicos do solo sobre a produtividade de trigo. Foram utilizadas técnicas de geoestatística como a construção de semivariogramas e confecção de mapas temáticos, produzidos por interpolação por krigagem de atributos físicos do solo, tais como: conteúdo gravimétrico de água no solo, porosidade total, grau de compactação e resistência do solo à penetração. Os dados experimentais foram obtidos em uma área de Latossolo Vermelho-Escuro, pertencente ao Núcleo Experimental de Engenharia Agrícola da Universidade Estadual do Oeste do Paraná - Campus de Cascavel. Os mapas dos atributos físicos do solo e da produtividade de trigo apresentaram um padrão de variabilidade, mostrando baixa produtividade nos locais de alto grau de compactação, baixa porosidade total e baixo conteúdo gravimétrico de água no solo e apresentando boa produtividade nos locais de baixa e média resistência do solo à penetração, médio e alto conteúdo de água no solo e baixo grau de compactação. Dentre os atributos físicos do solo estudados, a resistência do solo à penetração foi o atributo que melhor se correlacionou com a produtividade do trigo. Este trabalho possibilitou entender melhor a estrutura de dependência espacial das variáveis estudadas, bem com os conceitos e aplicação da Geoestatística.

Palavras chave: Autocorrelação, crosscorrelação, dependência espacial, krigagem, semivariograma

\begin{abstract}
The objective of this paper was to study the spatial variability influence of soil physical attributes in wheat yield. The geostatistics techniques used were semivariogram and contour maps produced by interpolation through usual kriging representing the spatial variability of the soil physical attributes: soil water content, soil total porosity, soil compaction degree and soil resistance to penetration.. Experimental data were at a Rhodic Ferralsol from the Agricultural Engineering Experimental Nucleus at UNIOESTE - Cascavel Campus. Soil physical attributes and wheat yield maps presented a variability standard regarding distribution in experimental area, showing low wheat yield where there was high soil compaction degree, low soil total porosity and low soil water content also presenting good wheat yield where low and medium soil resistance to penetration, medium and high soil water content and low soil compaction degree occurred. Among the studied soil physical attributes studied, soil resistance to penetration was the best attribute in correlation with wheat yield. The second best attribute was soil
\end{abstract}

1 Os dados utilizados neste artigo foram obtidos da dissertação de Mestrado em Engenharia Agrícola do primeiro autor.

2 Mestre em Engenharia Agrícola, Centro de Ciências Exatas e Tecnológicas - UNIOESTE. E-mail: natal@certto.com.br.

3 Docentes do Programa de Pós-Graduação em Engenharia Agrícola, Universidade Estadual do Oeste do Paraná, Cascavel, PR. E-mail:mhstavar@certto.com.bre mopazo@unioeste.br

* Autor para correspondência 
compaction degree. This paper enabled us to understand in a better way the spatial dependence structure of the studied attributes, as well as the concepts and geostatistics applications.

Key words: Autocorrelation, crosscorrelation, kriging, semivariogram, spatial dependence

\section{Introdução}

Um dos importantes fatores que deve ser levado em conta no planejamento da produção agrícola é a condição inicial do solo. Por isso, tanto a variabilidade espacial quanto a variabilidade temporal dos atributos do solo devem ser incorporadas aos procedimentos e tecnologias aplicados à agricultura (LI et al., 2002). Conhecendo a importância deste fato, já há alguns anos, agricultores estão adotando a Agricultura de Precisão, a qual tem como principal conceito a aplicação no local correto, no momento adequado, das quantidades de insumos necessárias à produção agrícola (BALASTREIRE, 2002). Para que isso ocorra de forma eficiente, é necessário utilizar ferramentas, tais como a Geoestatística, que permitam estabelecer modelos que descrevam a variabilidade espacial dos dados (CASSEL; WENDROTH; NELSEN, 2000).

Até recentemente, pesquisadores da área agronômica estudavam a variabilidade dos atributos do solo por meio da estatística clássica, a qual pressupõe que as observações de um dado atributo são independentes entre si, desconsiderando sua localização na área. Neste caso, os experimentos eram conduzidos para minimizar o impacto da variabilidade espacial, sendo ignorado o fato de que as observações podem ser espacialmente dependentes. Entretanto, tem sido constatado que observações adjacentes de alguns atributos do solo não são completamente independentes e que essa variabilidade espacial deve ser considerada na análise estatística dos dados (CARVALHO; DECHENS; DUFRANC, 2004).

A variabilidade espacial e temporal dos atributos dos solos ocorre em diferentes níveis, estando relacionada a fatores tais como: clima, relevo, ação de organismos, tempo, variação do material de origem nos processos genéticos de formação do solo e/ou efeitos de técnicas de manejo. Técnicas estatísticas, como: autocorrelogramas, crosscorrelogramas, semivariogramas, análise espectral, krigagem, cokrigagem, modelos de Espaço de Estados, têm sido utilizadas para estudar a variabilidade espacial dos atributos do solo, levando a um melhor entendimento dos processos de interação entre solo, planta e atmosfera (WENDROTH et al., 2001).

Pesquisadores como Diggle e Ribeiro Júnior (2007) têm demonstrado que, dentro um de certo domínio, as diferenças entre os valores de um atributo do solo podem ser expressas em função da distância de separação. Isto significa que os valores em locais mais próximos entre si são mais semelhantes do que aqueles tomados a maiores distâncias. Quando isto ocorre, os dados não podem ser tratados como independentes e um tratamento estatístico mais adequado é exigido (VIEIRA, 2000).

Quando se tem uma única amostragem por ponto e se deseja estimar valores para locais não amostrados, deve-se introduzir a restrição de que a variável regionalizada assume alguma forma de estacionaridade. Para Cressie (1993), a estacionaridade de ordem 2 é suficiente para o emprego da Geoestatística, permitindo a repetição do experimento mesmo que as amostras sejam coletadas em pontos diferentes, porque todas as amostras são consideradas pertencentes à população.

$\mathrm{Na}$ análise de variáveis regionalizadas, um dos conceitos mais empregados é o da função autocorrelação, definida como sendo a covariância entre valores amostrados, em relação à variância da população. A função autocorrelação leva à construção dos autocorrelogramas e apresenta o inconveniente de ser válida apenas se a hipótese de estacionaridade de ordem 2 for atendida, porque a função covariância necessita da média para estimação e esta não é mais homogênea para o conjunto dos dados. A função 
autocorrelação expressa a correlação linear de uma série de dados num espaço, quantificando a mudança de forte dependência para uma situação de independência. Outro conceito muito empregado é o da função semivariância, definida como a média entre variâncias de pares separados por um vetor distância e que leva à construção dos semivariogramas. Apresenta a grande vantagem de não exigir estacionaridade de ordem 2.

Quando a estacionaridade de ordem 2 é satisfeita, as funções de autocorrelação e de semivariância são equivalentes para caracterizar a correlação de uma variável espaçada de h. O autocorrelograma é padronizado no intervalo [-1,1], sendo o alcance (distância onde ocorre independência entre valores) determinado quando a curva atinge, aproximadamente, o valor zero. Já a curva do semivariograma, ao contrário do autocorrelograma, aumenta à medida que $\mathrm{h}$ cresce, atingindo o patamar quando a semivariância é, aproximadamente, igual à variância da população (VIEIRA, 2000).

Para a análise do grau de dependência espacial da variável, utiliza-se uma avaliação quantitativa da variabilidade espacial chamada de "coeficiente efeito pepita" $(\varepsilon)$, que é a relação percentual entre o efeito pepita $\left(\mathrm{C}_{0}\right)$ e o patamar $\left(\mathrm{C}_{1}+\mathrm{C}_{0}\right)$, ou seja:

$$
\varepsilon=\frac{C_{0}}{\left(C_{1}+C_{0}\right)} \times 100 .
$$

Quanto maior este coeficiente, menor a variabilidade espacial. O coeficiente de efeito pepita com valor até $25 \%$ é classificado como sendo de forte dependência espacial, os valores entre $25 \%$ e $75 \%$ como moderado e acima de $75 \%$ como tendo fraca dependência espacial (SOUZA et al., 1999).

$\mathrm{Na}$ maioria das vezes o interesse da análise geoestatística não se limita à obtenção de um modelo de dependência espacial, desejandose também predizer valores em pontos não amostrados. O interesse pode estar em um ou mais pontos específicos da área ou em obter uma malha de pontos interpolados que permitam visualizar o comportamento da variável através de um mapa de isolinhas ou de superfície. Para se obter esse maior detalhamento da área em estudo é necessária a aplicação de um método de interpolação, como a krigagem (VIEIRA, 2000).

O procedimento empregado na krigagem é semelhante ao de interpolação por médias móveis ponderada, com a diferença que os pesos são determinados a partir de uma análise espacial baseada no semivariograma experimental. $\mathrm{O}$ processo de krigagem diferencia-se dos demais métodos de interpolação pela forma de atribuição dos pesos, já que emprega uma "distância estatística" entre os pontos que expressa tanto a distância como a estrutura de variabilidade (semivariância ou covariância). Não apenas a distância dos vizinhos ao ponto a ser estimado é considerada, mas também as distâncias entre os próprios vizinhos entre si são consideradas na distribuição dos pesos. Assim, os vizinhos agrupados têm importância individual relativamente menor do que aqueles isolados (ISAAKS; SRIVASTAVA, 1989).

Neste trabalho, teve-se como objetivo estudar a variabilidade espacial dos atributos do solo: conteúdo gravimétrico de água no solo, porosidade total do solo, grau de compactação do solo, resistência do solo à penetração e analisar a influência dessa variabilidade na produtividade do trigo.

\section{Material e Métodos}

A aquisição de dados foi realizada no Núcleo Experimental de Engenharia Agrícola (NEEA), pertencente à UNIOESTE, Campus de Cascavel-PR. As coordenadas geográficas são: $24^{\circ} 54^{\prime}$ latitude Sul e 5331'57' longitude Oeste de Greenwich, altitude $750 \mathrm{~m}$. O clima da região é temperado mesotérmico e super-úmido, com precipitação anual em torno de $1920 \mathrm{~mm}$ e temperatura do ar com média anual em torno de $21^{\circ} \mathrm{C}$. O solo da região foi classificado como Latossolo Vermelho-Escuro distroférrico, substrato basalto e relevo suavemente ondulado (EMPRESA BRASILEIRA DE PESQUISA AGROPECUÁRIA - EMBRAPA, 1999). 
Os pontos de coletas de amostras foram delimitados em uma área de $311 \mathrm{~m}$ x $40 \mathrm{~m}$, na qual foram traçadas três transeções de $291 \mathrm{~m}$ cada. Nestas transeções foram delimitados 97 pontos de amostragem, com espaçamento de $3 \mathrm{~m}$ e com 10 $\mathrm{m}$ de espaçamento entre as transeções, conforme mostra a Figura 1. Os pontos foram demarcados com o auxílio de uma trena e georreferenciados com auxílio de GPS (Global Positioning System).

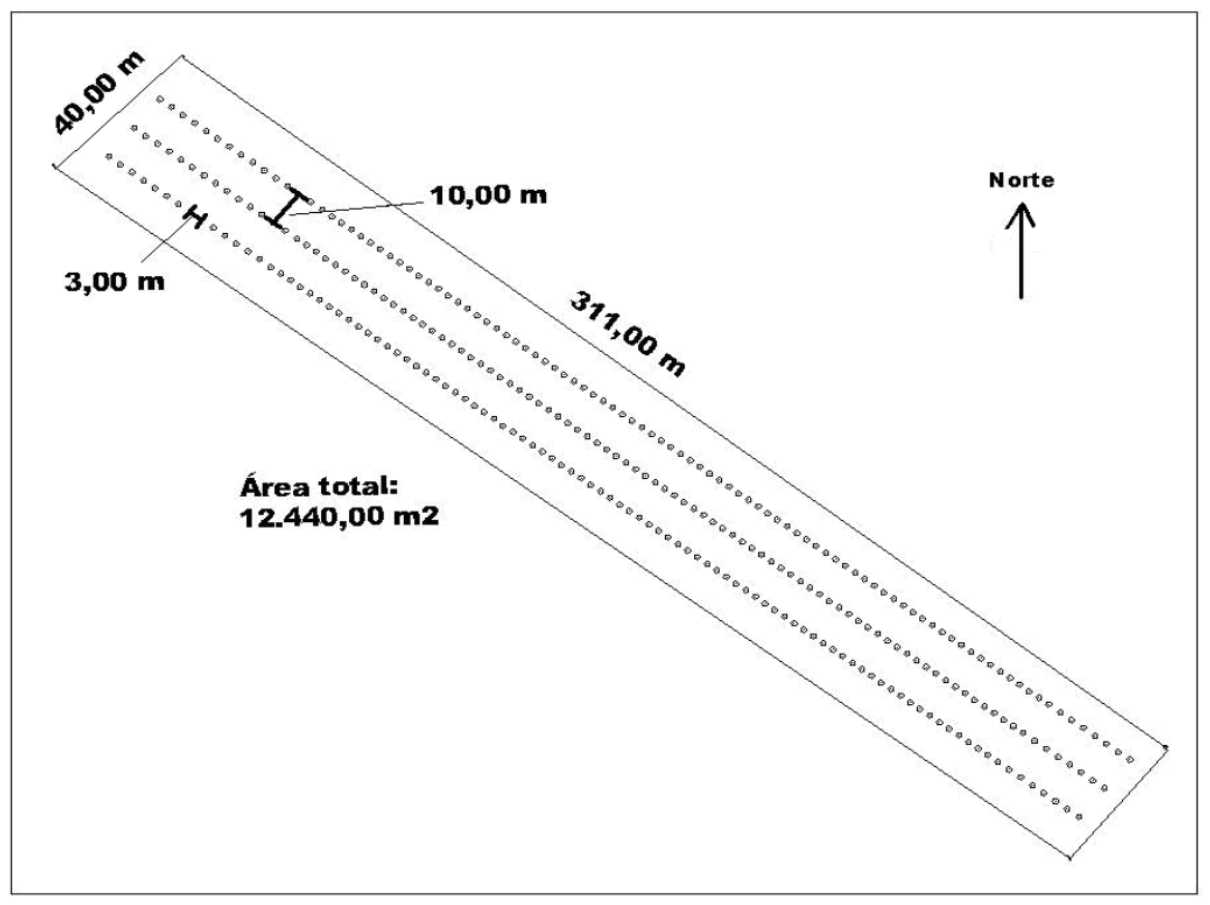

Figura 1. Pontos de coleta de amostras.

Os dados experimentais foram obtidos entre março e novembro de 2006. A variável resposta foi a produtividade do trigo e as variáveis independentes foram: conteúdo gravimétrico de água no solo, porosidade total do solo, grau de compactação do solo e resistência do solo à penetração. Todas as amostras e leituras foram coletadas na camada de solo entre $10-20 \mathrm{~cm}$.

Tanto o conteúdo gravimétrico de água no solo como a porosidade total do solo foram determinados conforme descreve o Manual de Métodos e Análises (EMBRAPA, 1997). Já o grau de compactação do solo foi obtido através da Equação 1: em que: GC é o grau de compactação (\%); $D s$ é a densidade do solo $\left(\mathrm{Mg} \mathrm{m}^{-3}\right)$ e $D_{M a x}$ é a densidade máxima do solo $\left(\mathrm{Mg} \mathrm{m}^{-3}\right)$.

Para se obter a densidade máxima do solo com teor de umidade ótimo de compactação, foi realizado em laboratório o Ensaio de Proctor Normal, através do qual foi obtida a curva de compactação do solo (DIAS JÚNIOR, 2000).

A Figura 2 apresenta a curva de compactação do solo, mostrando que o ponto de densidade máxima, $\left(\mathrm{D}_{\text {Max }}\right)$ é igual a $1,413 \mathrm{Mg} \mathrm{m}^{-3}$ para a umidade gravimétrica do solo de $0,321 \mathrm{~kg} \mathrm{~kg}^{-1}$.

$$
\mathrm{GC}=\frac{D s}{D_{\text {Max }}} \times 100
$$




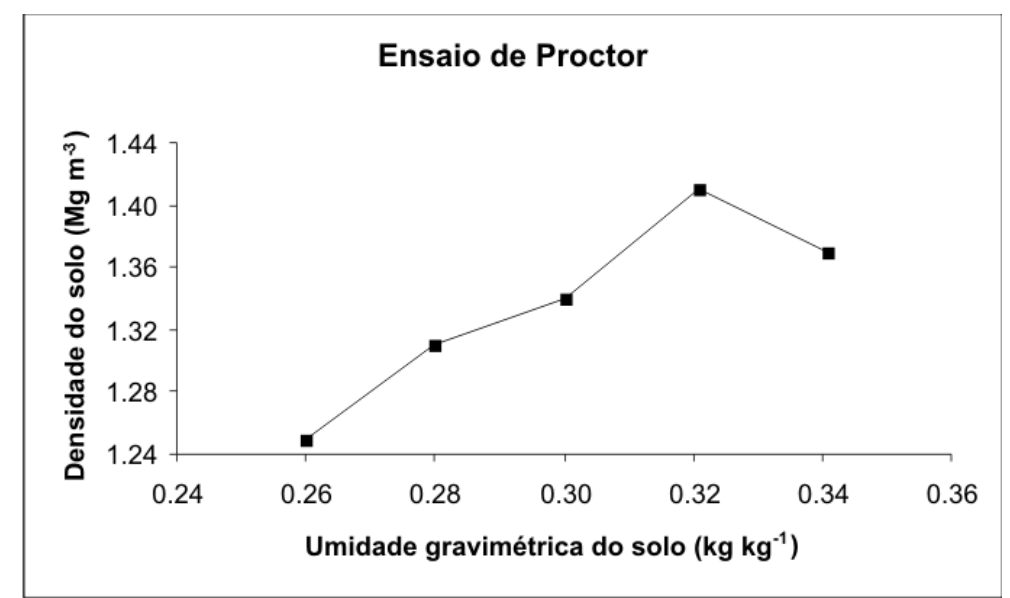

Figura 2. Curva de compactação do latossolo da área experimental.

A resistência do solo à penetração (RSP) foi determinada com o uso de um penetrômetro eletrônico, sendo o Índice de Cone obtido conforme norma da ASAE S313.1 (AMERICAN SOCIETY OF AGRICULTURAL ENGINEERS - ASAE, 1976), utilizando uma haste com cone de diâmetro de $12,83 \mathrm{~mm}$ e ângulo de $30^{\circ}$, sendo realizadas 4 repetições por ponto. A resistência do solo foi determinada 22 dias antes da colheita do trigo. O conteúdo gravimétrico de água no solo no período em que foram feitas as leituras da RSP foi em média $0,322 \mathrm{~kg} \mathrm{~kg}^{-1}$.

A produtividade do trigo foi determinada estabelecendo-se como unidade de análise em cada ponto de amostragem uma área de $1,0 \times 1,0 \mathrm{~m}$, delimitada com auxílio de um quadro de madeira, colocado com o ponto de referência no centro do quadrado. Os cachos de trigo foram colhidos manualmente, sendo em seguida descascados e limpos. A medida de massa foi realizada em uma balança digital com precisão de $0,01 \mathrm{~g}$ e os valores convertidos em $\mathrm{Mg} \mathrm{ha}^{-1}$.

$\mathrm{Na}$ análise exploratória dos dados e na construção de semivariogramas e mapas temáticos, utilizaramse os programas MINITAB 14 (MINITAB, 2003), SURFER 8 (GOLDEN SOFTWARE, 2002) e GS+ For Windows (GAMMA DESIGN SOFTWARE, 2000).

\section{Resultados e discussão}

$\mathrm{Na}$ Tabela 1 apresentam-se os resultados da estatística descritiva, da média das três transeções, para o conteúdo gravimétrico de água no solo (US), a porosidade total (PT), o grau de compactação do solo (GC), a resistência do solo à penetração (RSP) e a produtividade do trigo (PD).

A produtividade do trigo apresentou um valor médio de $0,331 \mathrm{Mg} \mathrm{ha}{ }^{-1}$, indicando uma produtividade muito baixa em relação à média regional, a qual foi de $1,280 \mathrm{Mg} \mathrm{ha}^{-1}$ em 2006 (COMPANHIA NACIONAL DO ABASTECIMENTO - CONAB, 2008). O baixo valor de produtividade pode ser explicado pelas condições climáticas desfavoráveis, pois o período outono-inverno de 2006 teve valores de chuva acumulada entre $40 \%$ a $60 \%$ do que seria o normal nesta época para a Região Oeste do Paraná. O coeficiente de variação (CV) de 25,29 \% indica alta variação dos dados em relação à média e mostra uma distribuição com alta heterogeneidade (GOMES, 1987).

Para resistência do solo à penetração, os dados observados tiveram a média de 2,42 $\mathrm{MPa}$, valor acima de 2,0 $\mathrm{MPa}$, o que pode ser considerado limitante ao crescimento radicular (SECCO et al. 2005). O coeficiente de variação (CV) igual a $11,16 \%$ indica pequena variação dos dados em 
relação à média e mostra uma distribuição com média homogeneidade.

Para o grau de compactação, os dados apresentaram média de $81,05 \%$. O coeficiente de variação $(\mathrm{CV})$ de 2,99 \% indica pequena variação em relação à média, mostrando homogeneidade nos dados.

Para porosidade total do solo, a média dos valores é de $55,9 \mathrm{~m}^{3} \mathrm{~m}^{-3}$, com um mínimo de $50,7 \mathrm{~m}^{3} \mathrm{~m}^{-3} \mathrm{e}$ com um máximo de $59,84 \mathrm{~m}^{3} \mathrm{~m}^{-3}$, que corresponde ao intervalo de ocorrência para solos argilosos, que é de $40 \mathrm{~m}^{3} \mathrm{~m}^{-3}$ a $60 \mathrm{~m}^{3} \mathrm{~m}^{-3}$ (AZEVEDO; DALMOLIN, 2004). Resultados semelhantes foram obtidos em áreas vizinhas por Milani (2005) e Bonini (2006). O coeficiente de variação $(\mathrm{CV})$ de 3,11 \% indica pequena variação dos dados em relação à média, mostrando a homogeneidade da distribuição.

Para o conteúdo gravimétrico de água no solo, com média dos dados de $30,02 \mathrm{~kg} \mathrm{~kg}^{-1}$, a pequena variação dos dados em relação à média, com coeficiente de variação (CV) de 4,87 \%, indica uma distribuição homogênea.

O teste de Kolmogorov-Smirnov indica que as cinco séries de dados possuem características de distribuição normal de probabilidade ao nível de $5 \%$ de significância, pois $\mathrm{KS}_{\text {crítico }}=0,150$.

Tabela 1. Estatística descritiva das variáveis estudadas.

\begin{tabular}{cccccccc}
\hline Variável & $\mathrm{N}$ & Média & Var & $\mathrm{CV}(\%)$ & Min & Max & KS \\
\hline US $\left(\mathrm{kg} \mathrm{kg}^{-1}\right)$ & 97 & 0,300 & 0,021 & 4,87 & 0,266 & 0,351 & 0,075 \\
PT $\left(\mathrm{m}^{3} \mathrm{~m}^{-3}\right)$ & 97 & 0,559 & 0,030 & 3,11 & 0,507 & 0,598 & 0,052 \\
GC $(\%)$ & 97 & 81,05 & 5,86 & 2,99 & 74,55 & 85,55 & 0,055 \\
RSP $(\mathrm{Mpa})$ & 97 & 2,42 & 0,073 & 11,16 & 1,88 & 2,96 & 0,054 \\
PD $\left(\mathrm{Mg} \mathrm{ha}^{-1}\right)$ & 97 & 0,331 & 0,007 & 25,29 & 0,193 & 0,582 & 0,081 \\
\hline
\end{tabular}

N: número de dados, Var: variância, CV: coeficiente de variação, Min: mínimo, Max: máximo, KS: teste de Kolmogorov-Smirnov ( $5 \%$ de significância), US: conteúdo gravimétrico de água no solo, PT: porosidade total do solo, GC: grau de compactação, RSP: resistência do solo à penetração, $\mathrm{PD}$ : produtividade do trigo, $\mathrm{KS}_{\text {crítico }}=0,150$ (5\% de significância).

Os gráficos boxplot das Figuras 3(a), (c) e (i) mostram que há um ponto na distribuição dos dados que pode ser considerado discrepante. Já os histogramas (b), (d), (f), (h) e (j) mostram curvas simétricas, indicando distribuição normal de dados.

Através da análise da Figura 4, verifica-se que o autocorrelograma (4a) apresenta autocorrelação até 14 lags, indicando que há dependência espacial até $42 \mathrm{~m}$ entre as observações do conteúdo gravimétrico de água no solo. O autocorrelograma (4b) apresenta autocorrelação até 12 lags, indicando que há dependência espacial até $36 \mathrm{~m}$ entre as observações da porosidade total do solo. O autocorrelograma (4c) apresenta autocorrelação até 4 lags, indicando que há dependência espacial até $12 \mathrm{~m}$ para o atributo grau de compactação do solo. No autocorrelograma (4d) verifica-se autocorrelação até 9 lags, fato que indica que há dependência espacial até $27 \mathrm{~m}$ entre as observações de resistência do solo à penetração e o autocorrelograma (4e) apresenta autocorrelação até 12 lags, indicando que há dependência espacial até $36 \mathrm{~m}$ entre as observações da produtividade do trigo. 
a)

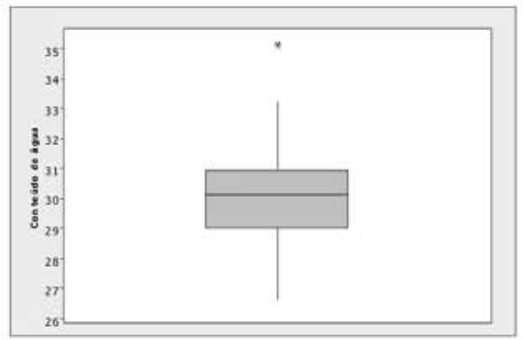

c)

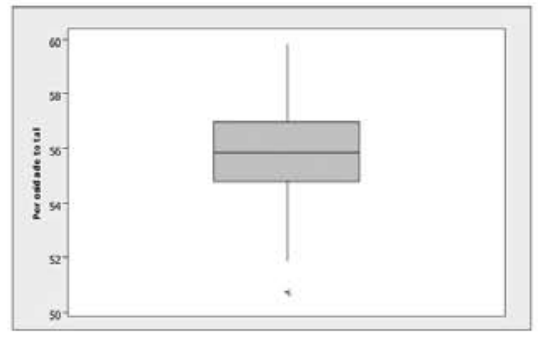

e)

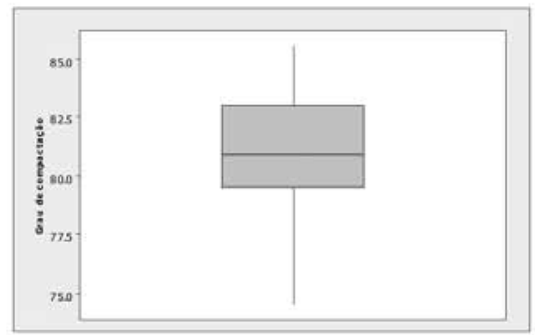

g)

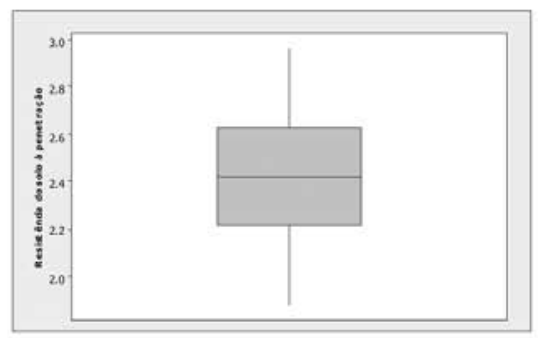

i)

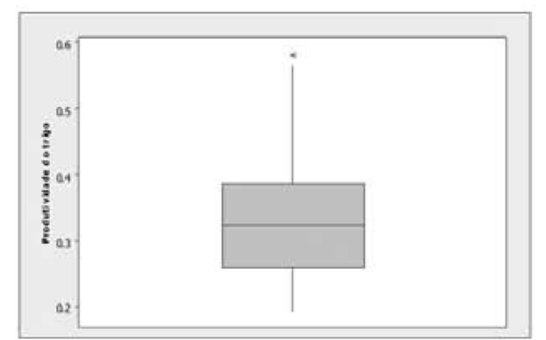

b)

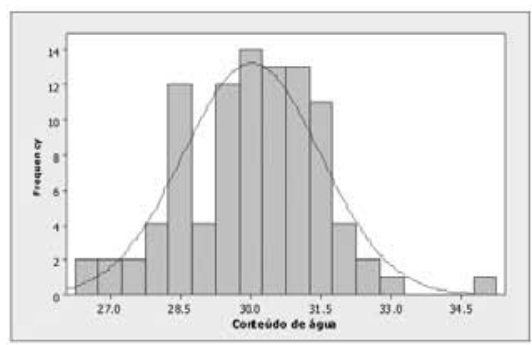

d)

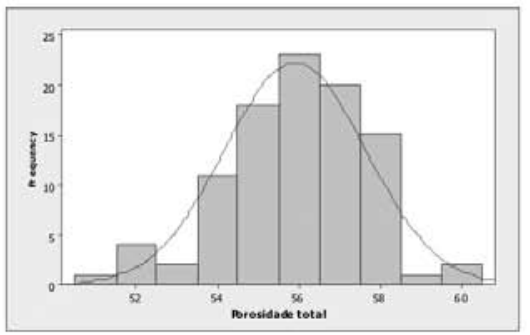

f)

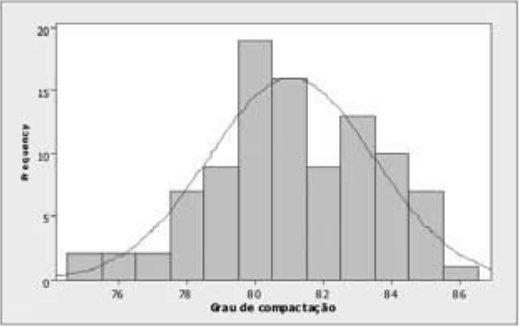

h)

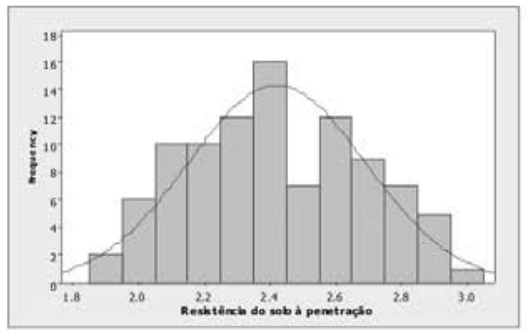

j)

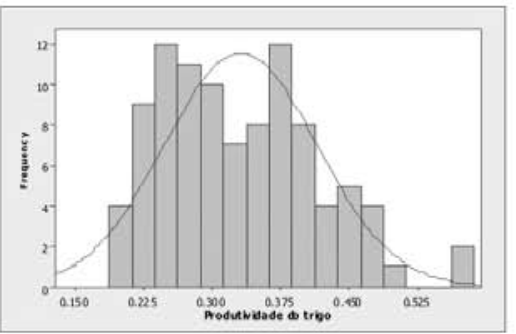

Figura 3. Boxplot e histograma com curva normal teórica do conteúdo gravimétrico de água no solo (a) e (b), porosidade total do solo $(\mathrm{c})$ e $(\mathrm{d})$, grau de compactação do solo $(\mathrm{e})$ e (f), resistência do solo à penetração $(\mathrm{g})$ e $(\mathrm{h})$ e produtividade do trigo (i) e (j). 
a)

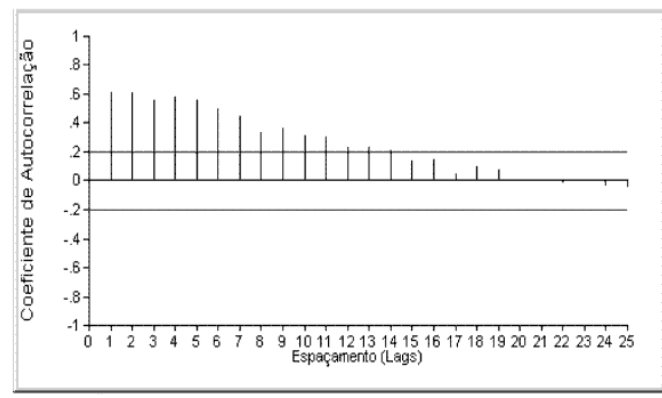

c)

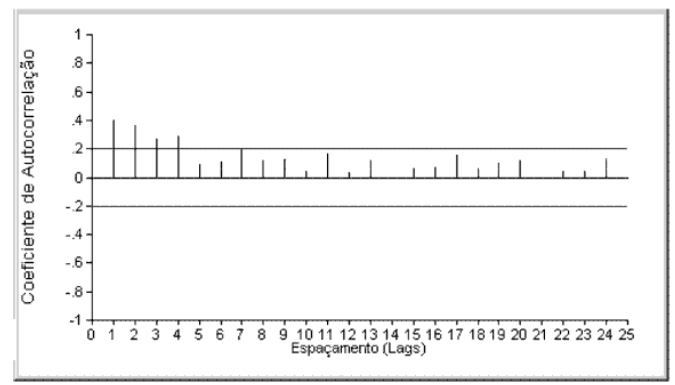

e)

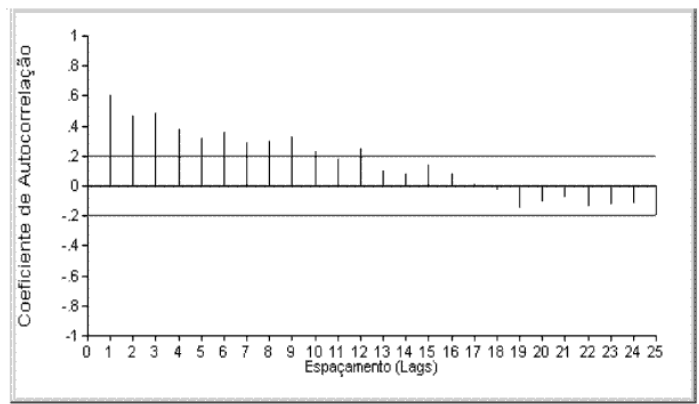

b)

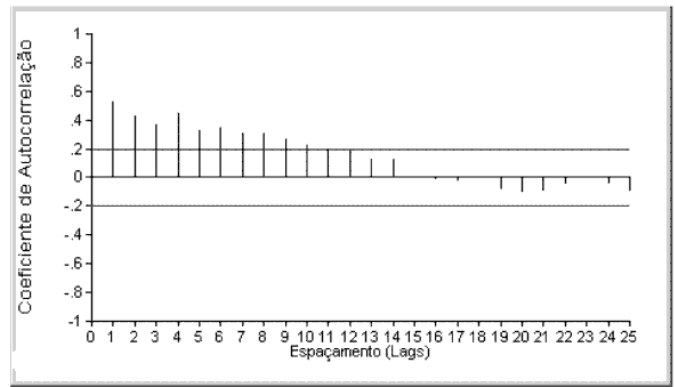

d)

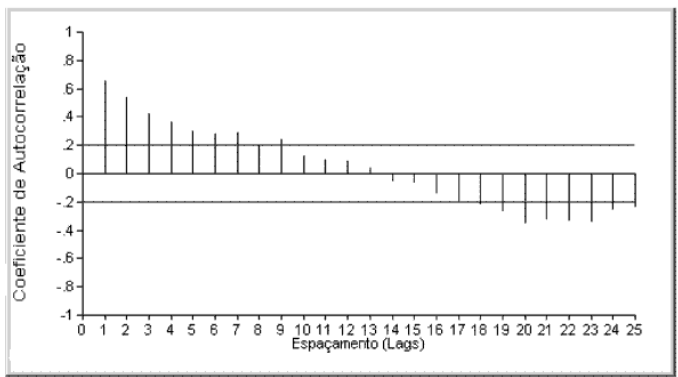

Figura 4. Função de autocorrelação do conteúdo gravimétrico de água no solo (a), da porosidade total do solo (b), do grau de compactação do solo (c), da resistência do solo à penetração (d) e da produtividade do trigo (e).

Os crosscorrelogramas apresentados nas Figuras $5 \mathrm{a}$ e $5 \mathrm{~b}$ mostram a correlação entre a produtividade do trigo com o conteúdo gravimétrico de água no solo e a porosidade total do solo. Como os gráficos não trazem valores acima ou abaixo dos limites de confiança a partir do ponto zero, estes atributos não apresentam correlação espacial entre si. O crosscorrelograma mostrado na Figura $5 \mathrm{c}$, correlacionando a produtividade do trigo com o grau de compactação do solo, indica que as duas variáveis são correlacionadas de forma inversamente proporcional até 3 lags, apresentando correlação espacial de até $9 \mathrm{~m}$. O crosscorrelograma $5 \mathrm{~d}$, correlacionando a produtividade do trigo com a resistência do solo à penetração, indica que as duas variáveis são correlacionadas até 8 lags, tendo correlação espacial até $24 \mathrm{~m}$. 
a)

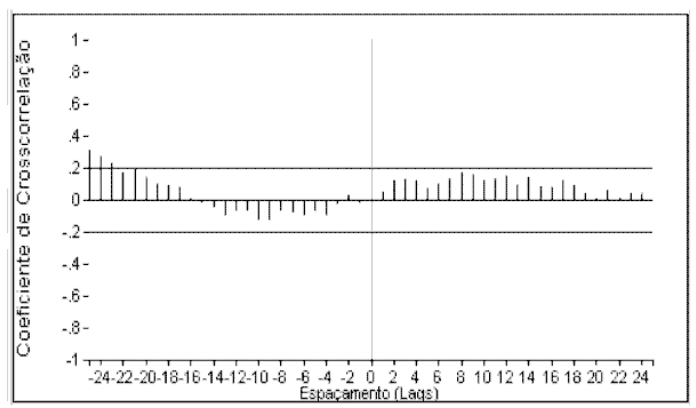

c)

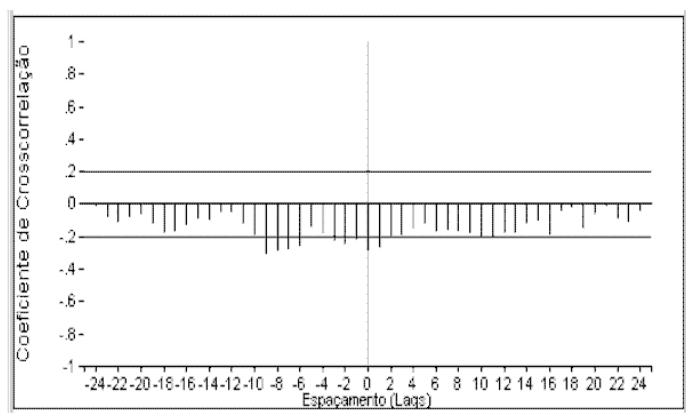

b)

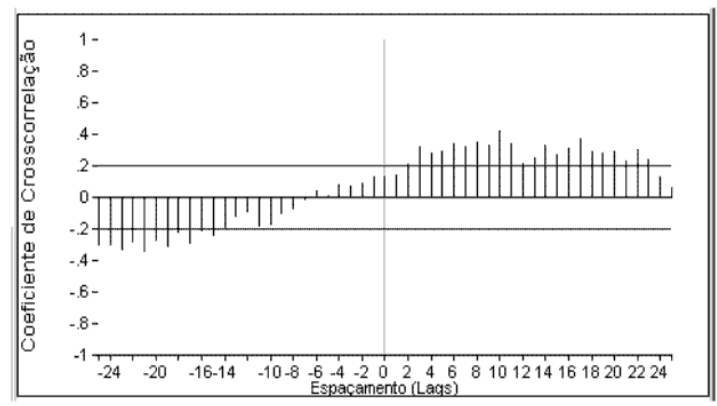

d)

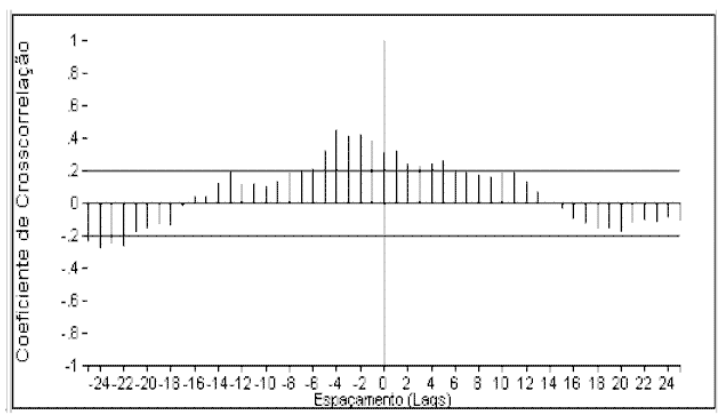

Figura 5. Função de crosscorrelação: da produtividade do trigo versus conteúdo gravimétrico de água no solo (a), da produtividade do trigo versus porosidade total do solo (b), da produtividade do trigo versus grau de compactação do solo $(\mathrm{c})$ e da produtividade do trigo versus resistência do solo à penetração $(\mathrm{d})$.

A Figura 6 apresenta os semivariogramas: (a) do conteúdo gravimétrico de água no solo, (b) da porosidade total do solo, (c) do grau de compactação do solo, (d) da resistência do solo à penetração e (e) da produtividade do trigo.

Todos os semivariogramas acima mostrados indicam estacionaridade de ordem 2 para a respectiva variável, pois cada um apresenta patamar claro e bem definido. Por meio da construção dos semivariogramas foi constatado que a distribuição dos dados em estudo é um fenômeno isotrópico, pois os semivariogramas construídos para as diferentes direções $\left(0^{\circ}, 45^{\circ}, 90^{\circ}\right.$ e $\left.135^{\circ}\right)$ não apresentam diferenças relevantes, indicando que a área de influência é circular. Os semivariogramas das Figuras 6a para o conteúdo gravimétrico de água no solo com alcance $\mathrm{a}=120,90 \mathrm{~m}$ e $\varepsilon=49,9 \%, 6 \mathrm{~b}$ para a porosidade total com a $=72,90 \mathrm{~m} \mathrm{e} \varepsilon=47,5$ $\%, 6 \mathrm{~d}$ para a resistência do solo à penetração com a $=46,70 \mathrm{~m}$ e $\varepsilon=45,0 \%$ e 6 e para a produtividade do trigo com alcance $\mathrm{a}=45,60 \mathrm{~m}$ e $\varepsilon=27,3 \%$ indicam dependência espacial moderada. Jáo semivariograma $6 \mathrm{c}$, relativo ao grau de compactação, com alcance a $=27,60 \mathrm{~m}$ e $\varepsilon=24,2 \%$, indica forte dependência espacial para a área e espaçamentos utilizados na amostragem. 
a)

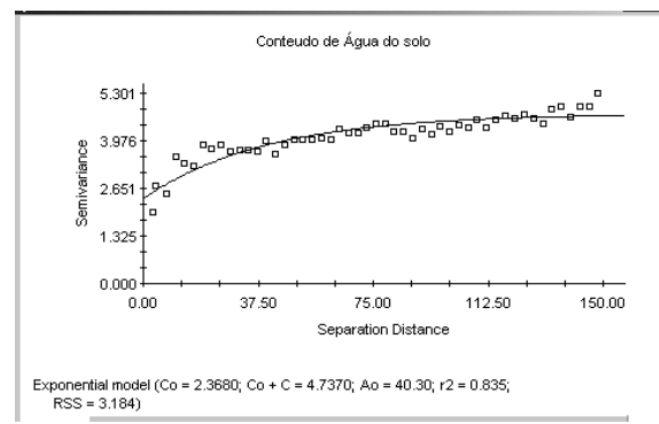

c)

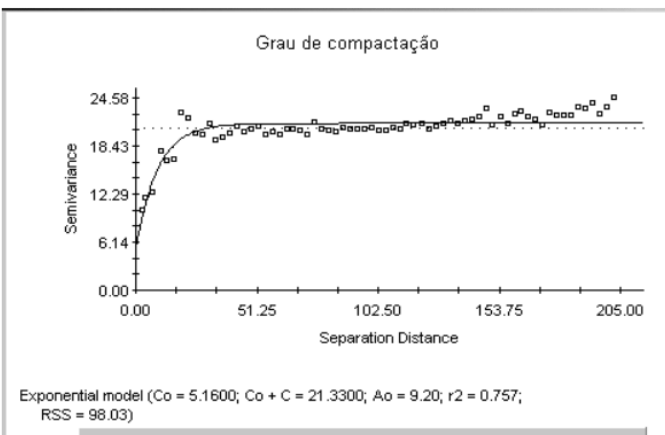

e)

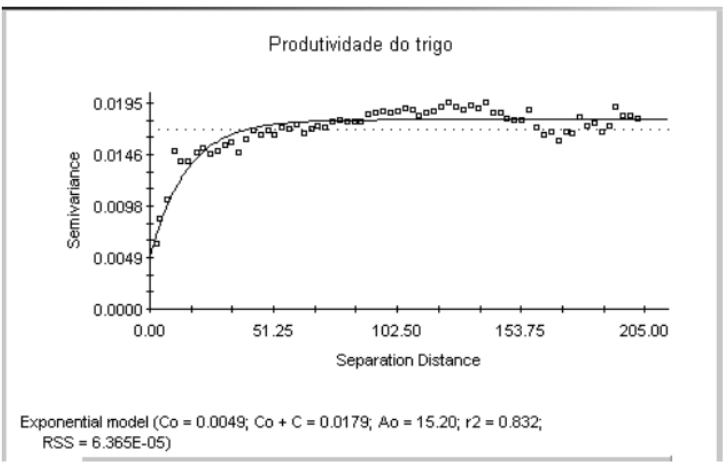

b)

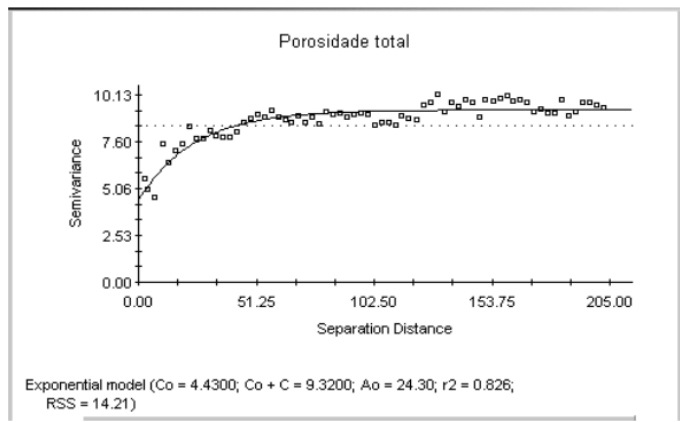

d)

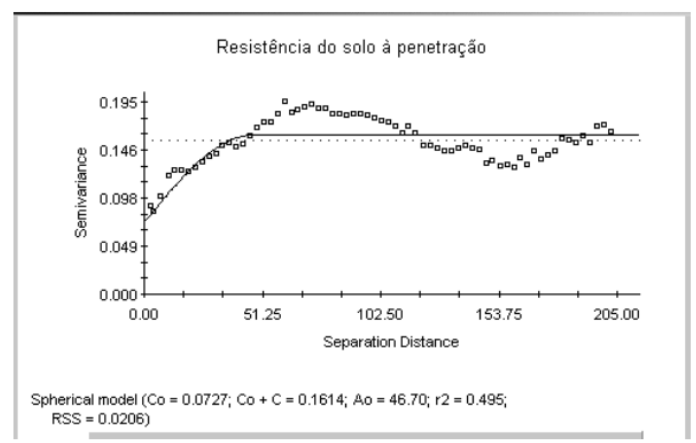

Figura 6. Semivariogramas experimental e teórico: (a) do conteúdo gravimétrico de água no solo, (b) da porosidade total do solo, (c) do grau de compactação do solo, (d) da resistência do solo à penetração e (e) da produtividade do trigo. 
Para a análise do grau de dependência espacial das variáveis foi utilizada a relação "coeficiente efeito pepita", cujos resultados são apresentados na Tabela 2. Os alcances semelhantes apresentados pela produtividade do trigo e pela resistência do solo à penetração são explicados pela correlação verificada entre as duas variáveis, a qual também explica as semelhanças entre os semivariogramas (CICHOTA; LIER; ROJAS, 2003). O fato de o modelo exponencial modelar todos os semivariogramas indica que o patamar é atingido de forma assintótica, diferentemente do que ocorre com o modelo esférico, no qual a estabilização é atingida no valor do alcance (VIEIRA, 2000).

Tabela 2. Semivariogramas experimentais das variáveis em estudo.

\begin{tabular}{clllll}
\hline Variável & Modelo & $\mathbf{C}_{\mathbf{0}}$ & $\mathbf{C}_{\mathbf{0}}+\mathbf{C}_{\mathbf{1}}$ & $\mathbf{a}(\mathbf{m})$ & $\mathbf{\varepsilon}(\mathbf{\%})$ \\
\hline US & Exponencial & 2,362 & 4,737 & 120,90 & 49,9 \\
PT & Exponencial & 4,43 & 9,320 & 72,90 & 47,5 \\
GC & Exponencial & 5,18 & 21,330 & 27,60 & 24,2 \\
RSP & Esférico & 0,0727 & 0,161 & 46,70 & 45,0 \\
PD & Exponencial & 0,0049 & 0,0179 & 45,60 & 27,3 \\
\hline
\end{tabular}

$\mathrm{C}_{0}$ : efeito pepita, $\mathrm{C}_{0}+\mathrm{C}_{1}$ : patamar, $a$ : alcance $(\mathrm{m})$, $\varepsilon$ : coeficiente efeito pepita (\%), US: conteúdo gravimétrico de água no solo, PT: porosidade total, DS: densidade do solo, GC: grau de compactação, RSP: resistência do solo à penetração, PD: produtividade do trigo.

Note-se que a diferença fundamental entre as técnicas geoestatisticas aqui empregadas é o fato que, enquanto os autocorrelogramas mostram apenas a correlação espacial significativa, já os semivariogramas determinam os alcances e o patamar. Como o semivariograma é uma ferramenta mais completa, as conclusões se baseiam nos resultados encontrados a partir de sua análise, enquanto o autocorrelograma atua como suporte adicional.

Os mapas temáticos mostrados na Figura 7 foram construídos utilizando-se os 291 pontos amostrais, com suas coordenadas geográficas, por meio do método da krigagem, através das estruturas de dependência espacial obtidas dos semivariogramas. $\mathrm{O}$ polígono retangular em diagonal mostra o perímetro da área experimental.

A Figura 7a mostra o mapa de variabilidade espacial do conteúdo gravimétrico de água no solo, com dependência espacial moderada. O local com os mais baixos valores do conteúdo de água apresentou também baixa produtividade e está localizado numa baixada ao norte do talhão.

A Figura $7 \mathrm{~b}$ apresenta o mapa de variabilidade espacial da porosidade total do solo que mostra dependência espacial moderada. Nota-se que a porosidade total é maior nos dois extremos do talhão, nos pontos onde o grau de compactação é menor e a produtividade é média.

A Figura 7c apresenta o mapa de variabilidade espacial do grau de compactação do solo, mostrando forte dependência espacial. O mapa temático mostra que, na área onde ocorreu o menor grau de compactação, o conteúdo gravimétrico de água apresentou o maior valor e a produtividade também foi a maior.

A Figura 7d mostra o mapa temático de variabilidade espacial da resistência do solo à penetração, com dependência espacial moderada. Nos locais onde foram encontrados os menores valores da RSP, a produtividade do trigo também apresentou os menores valores. Já nos locais onde 
a RSP foi alta, ocorreram baixa taxa de umidade gravimétrica e média porosidade total do solo.

A Figura 7e apresenta o mapa de variabilidade espacial da produtividade do trigo, o qual mostra que a região central da área de trabalho foi o local onde ocorreu a menor produtividade. A região que apresentou a maior produtividade teve o mais baixo grau de compactação, média taxa de porosidade total e média RSP, estando próxima à cabeceira do talhão, ao sul. (a)

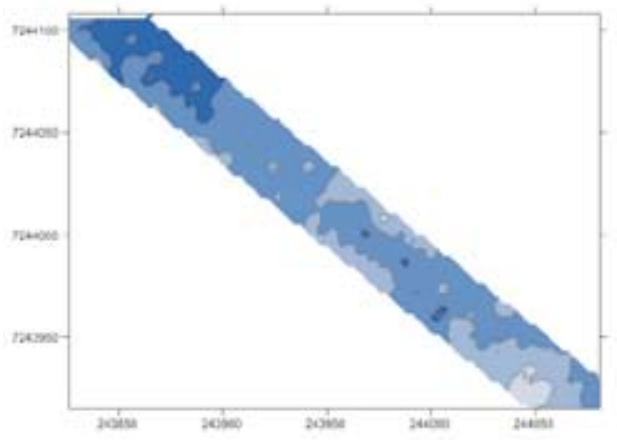

(c)

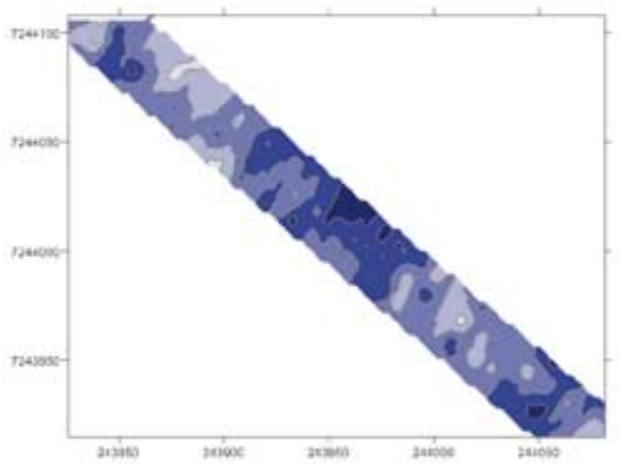

(e)

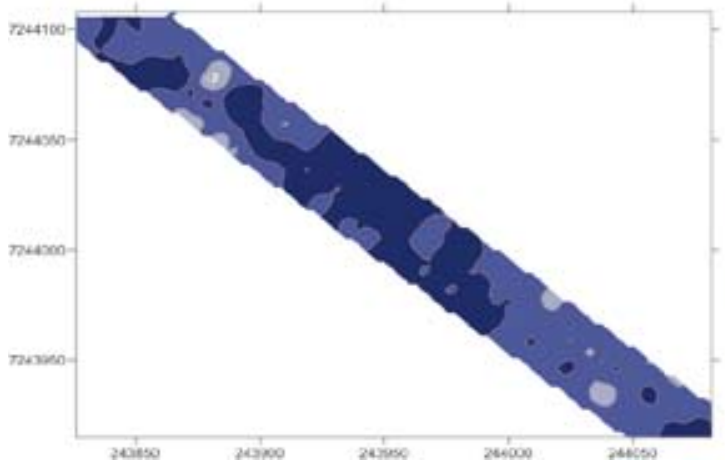

(b)

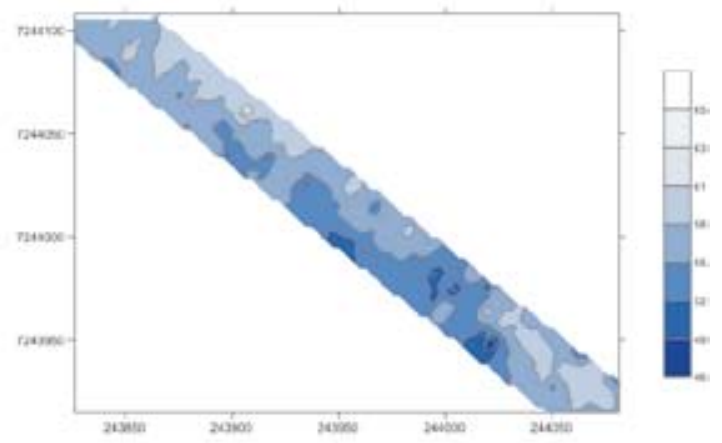

(d)
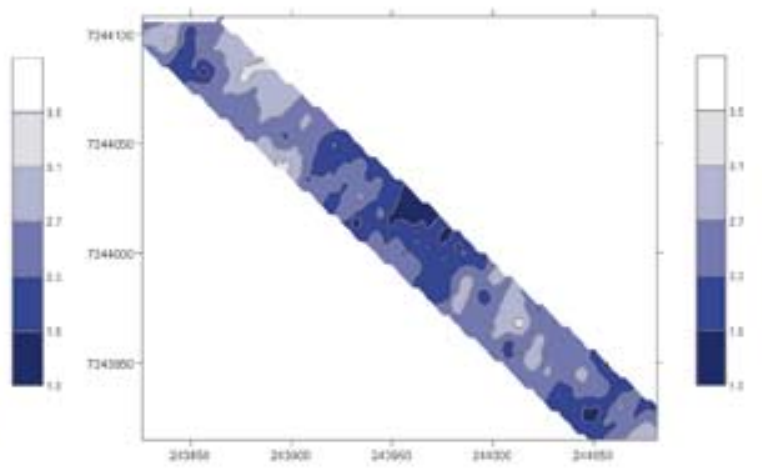

Figura 7. Mapas temáticos do conteúdo gravimétrico de água no solo (a) $\left(\mathrm{kg} \mathrm{kg}^{-1}\right)$, da porosidade total do solo (b) $\left(\mathrm{m}^{3} \mathrm{~m}^{-3}\right)$, do grau de compactação do solo $(\mathrm{c})(\%)$, da resistência do solo à penetração (d) (MPa) e da produtividade do trigo (e) $\left(\mathrm{Mg} \mathrm{ha}^{-1}\right)$. 


\section{Conclusões}

Por meio da análise geoestatística foi constatado que a distribuição dos dados em estudo é um fenômeno isotrópico, o que significa que os semivariogramas construídos para as diferentes direções não apresentam diferenças relevantes e um mesmo modelo é adequado para todos eles, indicando que a área de influência é circular.

A análise geoestatística dos atributos: conteúdo gravimétrico de água no solo, porosidade total do solo, resistência do solo à penetração e produtividade do trigo mostrou dependência espacial moderada. Já o grau de compactação do solo apresentou forte dependência espacial para a área e espaçamentos utilizados na amostragem. Esta dependência espacial apresentada nos semivariogramas foi constatada também pelos autocorrelogramas destes atributos.

Nos crosscorrelogramas, foi verificada a crosscorrelação somente entre os atributos grau de compactação do solo e resistência do solo à penetração com a produtividade do trigo. Isto significa que estes atributos apresentam dependência espacial em relação à produtividade do trigo.

Os mapas dos atributos físicos do solo $\mathrm{e}$ da produtividade de trigo mostraram baixa produtividade onde havia alto grau de compactação, baixa porosidade total e baixo conteúdo de água no solo e apresentaram boa produtividade onde ocorreu baixa e média resistência do solo à penetração, médio e alto conteúdo de água no solo e baixo grau de compactação.

Dentre os atributos físicos do solo estudados, a resistência do solo à penetração foi o atributo que melhor se correlacionou com a produtividade do trigo. O segundo melhor atributo foi o grau de compactação do solo.

\section{Referências}

AMERICAN SOCIETY OF AGRICULTURAL ENGINEERS - ASAE. Soil cone penetrometer. In: (Ed). Agricultural engineers handbook. Saint Joseph: Soil Cone Penetrometer, 1976. p. 368-369. (ASAE Rec., 313.1).

AZEVEDO, A. C.; DALMOLIN, R. S. D. Solos e ambiente: uma introdução. Santa Maria: Pallotti, 2004.

BALASTREIRE, L. A. Avanços na agricultura de precisão no Brasil no periodo de 1999-2001. Piracicaba: Edusp, 2002.

BONINI, A. K. Compactação de um Latossolo vermelho distroférrico sob sistema plantio direto e rendimento de grãos de trigo, soja e milho. 2006. Dissertação. (Mestrado em Engenharia Agrícola) - Universidade Estadual do Oeste do Paraná, Cascavel.

CARVALHO, J. R. P.; DECHENS, S. C. F.; DUFRANC, G. Variabilidade espacial da agregação do solo avaliada pela geometria fractal e geoestatística. Revista Brasileira de Ciência do Solo, Viçosa, v. 28, n. 1, p. 1-9, 2004.

CASSEL, D. K.; WENDROTH, O.; NIELSEN, D. R. Assessing spatial variability in an agricultural experiment station field: opportunities arising from spatial dependence. Agronomy Journal, Madison, v. 92, n. 4 , p. $706-714,2000$.

CICHOTA, R.; LIER, Q. J.; ROJAS, C. A. L. Variabilidade espacial da taxa de infiltração em argissolo vermelho. Revista Brasileira de Ciência do Solo, Viçosa, v. 27, n. 5, p. 789-798, 2003.

COMPANHIA NACIONAL DE ABASTECIMENTO - CONAB. Ministério da Agricultura, Pecuária e Abastecimento. Trigo - Brasil: Série Histórica de Produção. Disponível em: <http://www.conab.gov.br/ conaweb/>. Acesso em: 14 maio 2008.

CRESSIE, N. Statistics for spatial data. New York: John Willey \& Sons, 1993.

DIAS JÚNIOR, M. S. Compactação do solo. In: NOVAIS, R. F.; ALVAREZ, V. H.; SCHAEFER, C. E. G. R. Tópicos em ciência do solo. Viçosa: Sociedade Brasileira de Ciência do Solo, 2000. v. 1, p. 55-89.

DIGGLE, P. J.; RIBEIRO JÚNIOR, P. J. Model - based geostatistics. New York: Springer, 2007. 
EMPRESA BRASILEIRA DE PESQUISA AGROPECUÁRIA - EMBRAPA. Centro Nacional de Pesquisa de Solos. Ministério da Agricultura e do Abastecimento. Sistema brasileiro de classificação de solos. Brasília: EMBRAPA, 1999.

Centro Nacional de Pesquisa de Solos. Ministério da Agricultura e do Abastecimento. Manual de métodos de análise do solo. Rio de Janeiro: EMBRAPA, 1997.

GAMMA DESIGN SOFTWARE. GS+ for Windows. Demonstration version. Michigan: Gamma Design Software, 2000.

GOLDEN SOFTWARE. Surfer for Windows version 8.0. Colorado: Golden, 2002.

GOMES, F. P. Curso de estatística experimental. 12. ed. Piracicaba: Nobel, 1987.

ISAAKS, E. H.; SRIVASTAVA, R. M. an introduction to applied geostatistics. Oxford: Oxford University Press, 1989.

LI, H.; LASCANO, R. J.; BOOKER, J.; WILSON, L. T.; BRONSON, K. F.; SEGARRA, E. State-space description of heterogeneity: water and nitrogen use in cotton. Soil Science Society of America Journal, Madison, v. 66, n. 2, p. 585-595, 2002.

MILANI, D. I. C. Variabilidade espacial da infiltração de água e de atributos físicos em um Latossolo sob sistema plantio direto. 2005. Dissertação (Mestrado em Engenharia Agrícola) - Universidade Estadual do Oeste do Paraná, Cascavel.
MINITAB. Minitab for Windows - Version 14 PA. Pensilvânia: State College, 2003.

SECCO, D.; ROS, C. O. D.; SECCO, J. K.; FIORIN, J. E. Atributos físicos e produtividades de culturas em um Latossolo Vermelho argiloso sob diferentes sistemas de manejo. Revista Brasileira de Ciência do Solo, Viçosa, v. 29, n. 3, p. 407-414, 2005.

SOUZA, E. G.; JOHANN, J. A.; ROCHA, J. V.; RIBEIRO, S. R. A.; SILVA, M. S.; URIBE-OPAZO, M. A.; MOLIN, J. P.; OLIVEIRA, E. F.; NÓBREGA, L. H. P. Variabilidade espacial dos atributos químicos do solo em um latossolo roxo distrófico na região de Cascavel - PR. Revista da Sociedade Brasileira de Engenharia Agrícola, Jaboticabal, v. 8, n. 3, p. 80-92, 1999.

VIEIRA, S. R. Geoestatística em estudos de variabilidade espacial do solo. In: NOVAIS, R. F.; ALVAREZ, V. H.; SCHAEFER, C. E. G. R. (Ed.) Tópicos em ciência do solo. Viçosa: Sociedade Brasileira de Ciência do Solo, 2000. v. 1, p. 1-53.

WENDROTH, O.; JÜRSCHIK, P.; KERSEBAUM, K. C.; REUTER, H.; VAN KESSEL, C.; NIELSEN, D. R. Identifying, understanding and describing spatial processes in agricultural landscapes - four case studies. Soil \& Tillage Research, Oxford, v. 58, n. 3-4, p. 113127, 2001. 